\title{
Trends of Repetitive Transcranial Magnetic Stimulation From 2009 to 2018: A Bibliometric Analysis
}

\author{
Kang-Yong Zheng ${ }^{1,2+}$, Guang-Yan Dai ${ }^{3+}$, Yue Lan ${ }^{4 *}$ and Xue-Qiang Wang ${ }^{1,5 *}$ \\ ${ }^{1}$ Department of Sport Rehabilitation, Shanghai University of Sport, Shanghai, China, ${ }^{2}$ The Fifth Clinical College, Guangzhou \\ Medical University, Guangzhou, China, ${ }^{3}$ Department of Rehabilitation Medicine, The First Affiliated Hospital, Sun Yat-sen \\ University, Guangzhou, China, ${ }^{4}$ Department of Rehabilitation Medicine, Guangzhou First People's Hospital, School of \\ Medicine, South China University of Technology, Guangzhou, China, ${ }^{5}$ Department of Rehabilitation Medicine, Shanghai \\ Shangti Orthopaedic Hospital, Shanghai, China
}

OPEN ACCESS

Edited by: loan Opris,

University of Miami, United States

Reviewed by: Estate M. Sokhadze, University of South Carolina,

United States Kim Drnec,

United States Army Research Laboratory, United States

*Correspondence:

Yue Lan

bluemooning@163.com Xue-Qiang Wang qiang897@163.com

these authors have contributed equally to this work

Specialty section:

This article was submitted to Neural Technology,

a section of the journal Frontiers in Neuroscience

Received: 03 October 2019 Accepted: 27 January 2020 Published: 26 February 2020

Citation:

Zheng K-Y, Dai G-Y, Lan Y and Wang X-Q (2020) Trends of Repetitive Transcranial Magnetic Stimulation From 2009 to 2018: A Bibliometric Analysis. Front. Neurosci. 14:106 doi: 10.3389/fnins.2020.00106
Repetitive transcranial magnetic stimulation (rTMS) technology, which is amongst the most used non-invasive brain stimulation techniques currently available, has developed rapidly from 2009 to 2018. However, reports on the trends of rTMS using bibliometric analysis are rare. The goal of the present bibliometric analysis is to analyze and visualize the trends of rTMS, including general (publication patterns) and emerging trends (research frontiers), over the last 10 years by using the visual analytic tool CiteSpace V. Publications related to rTMS from 2009 to 2018 were retrieved from the Web of Science (WoS) database, including 2,986 peer-reviewed articles/reviews. Active authors, journals, institutions, and countries were identified by WoS and visualized by CiteSpace V, which could also detect burst changes to identify emerging trends. GraphPad Prism 8 was used to analyze the time trend of annual publication outputs. The USA ranked first in this field. Pascual-Leone A (author A), Fitzgerald PB (author B), George MS (author C), Lefaucheur JP (author D), and Fregni F (author E) made great contributions to this field of study. The most prolific institution to publish rTMS-related publications in the last decade was the University of Toronto. The journal Brain Stimulation published most papers. Lefaucheur et al.'s paper in 2014, and the keyword "sham controlled trial" showed the strongest citation bursts by the end of 2018, which indicates increased attention to the underlying work, thereby indicating the research frontiers. This study reveals the publication patterns and emerging trends of rTMS based on the records published from 2009 to 2018. The insights obtained have reference values for the future research and application of rTMS.

\section{Keywords: rTMS, frontiers, bibliometrics, citation burst, Web of Science, CiteSpace}

\section{INTRODUCTION}

Repetitive transcranial magnetic stimulation (rTMS) is a variant of transcranial magnetic stimulation (TMS) that can be applied to the modulation of corticospinal excitability from outside the skull via a time-varying magnetic field to generate electric current in the underlying brain tissue, leading to neuronal depolarization (Maeda et al., 2000; Klooster et al., 2016; Barker and Shields, 2017). rTMS is the most widely used non-invasive brain stimulation technique currently available (George and Aston-Jones, 2010; Miniussi et al., 2013; Cirillo et al., 2017; Lowe et al., 2017; Lucena et al., 2019). Numerous studies have investigated the effects and mechanisms underlying various 
rTMS protocols, which remain incompletely understood (Fitzgerald et al., 2006b; Boonzaier et al., 2018; Zorzo et al., 2019). Low-frequency $(\leq 1.0 \mathrm{~Hz})$ rTMS usually reduces cortical excitability, whereas high-frequency $(>1.0 \mathrm{~Hz})$ rTMS (HFrTMS) raises excitability (Maeda et al., 2000; Rossini et al., 2015). Over the last decade, rTMS has been explored as a tool for the treatment of various neuropsychiatric conditions, including, but not limited to, depression, neuropathic pain, stroke, epilepsy, anxiety, schizophrenia, Parkinson's disease, obsessive compulsive disorder, and autism (Pascual-Leone et al., 1996; Hummel and Cohen, 2006; Hao et al., 2013; Hosomi et al., 2013). Combined strategies, i.e., combination of rTMS with neuroimaging techniques, medication, physiotherapy, or psychotherapy, have also been investigated to optimize the use of the technique (Reithler et al., 2011; Dayan et al., 2013; Kwon et al., 2016; Jansen et al., 2019; Terranova et al., 2019).

Bibliometrics is a quantitative method for analyzing literature in analytical science and assessing trends in research activities over time (Oelrich et al., 2007; Ellegaard and Wallin, 2015; Thompson and Walker, 2015). Bibliometric studies have been used in various areas, such as medical big data, pain, cognitive function, and neuroimaging, in recent years (Yeung et al., 2017; Liao et al., 2018; Wang et al., 2019; Zheng and Wang, 2019). A considerable number of scholars and academic journals have focused on publishing rTMS research over the last decade. However, reports of trends of rTMS using bibliometric analysis are rare.

This study conducts a bibliometric analysis of rTMS on the basis of records published from 2009 to 2018 to identify the publication patterns and emerging trends of this technique and gain new insights to guide future research and application.

\section{MATERIALS AND METHODS}

\section{Source of Data and Search Strategy}

Published papers were retrieved via a topic search of the Science Citation Index Expanded (SCI-EXPANDED) index of the WoS database on 6 April 2019. The following search terms were used: topic $=$ ("repetitive transcranial magnetic stimulation" or "rTMS"), index = SCI-EXPANDED and time span =2009-2018.

\section{Inclusion Criteria}

The inclusion criteria are shown in Figure 1. A record was considered relevant if "repetitive transcranial magnetic stimulation" or "rTMS" was found in its title, abstract, or keywords. Only articles and reviews were included; other document types, such as meeting abstracts and letters, were excluded. In addition, the publication language was restricted to English, and no species limitations were set. A total of 2,986 records published during the period 2009-2018 met the inclusion criteria.

\section{Analytical Methods}

WoS provides detailed features of publications, such as number of papers, citations, citations per paper, essential science indicator (ESI) top papers, and Hirsch index (H-index). The number of papers reflects the research productivity. Citations measure the overall impact of the scientific output of a researcher, while the

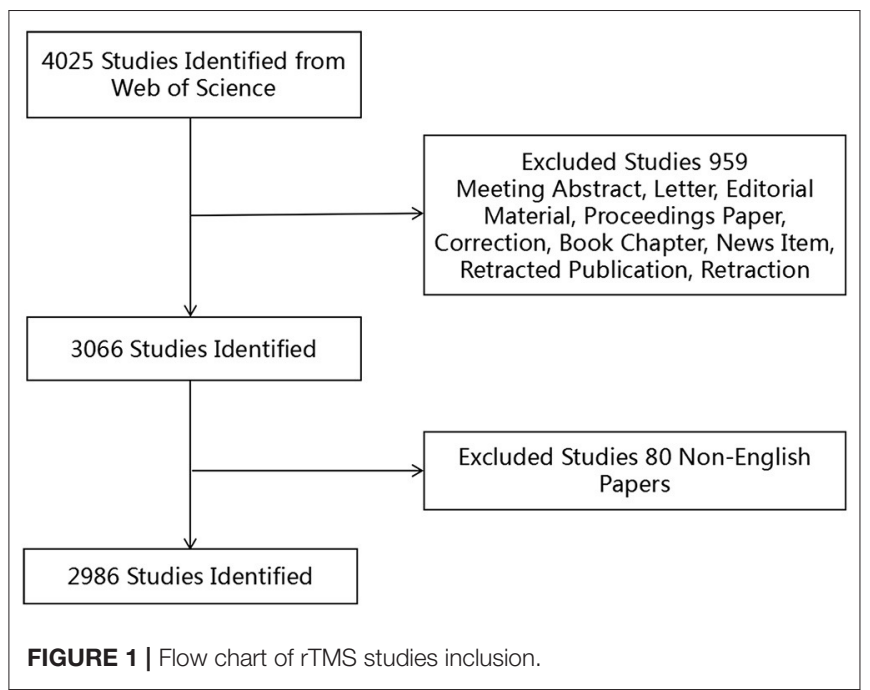

number of citations per paper measures the average impact. ESI top papers represent the most cited papers, including the hot papers and highly cited papers over the last 2 and 10 years, respectively (Fitzpatrick, 2005; Fu et al., 2011). The H-index is defined that a scientist has published $h$ papers that have each been cited at least $\mathrm{h}$ times (if the $\mathrm{H}$-index of a given individual is 10 , this means that he published at least 10 papers and each of these 10 papers was cited at least 10 times) (Hirsch, 2005; McLean, 2019; Wang et al., 2019). The H-index evaluates the broad impact of the cumulative scientific publications of an author or country (Alonso et al., 2009; Bornmann and Daniel, 2009; da Silva and Dobranszki, 2018). Finally, impact factor (IF), according to Journal Citation Reports (2019), indicates the impact of journals.

CiteSpace V, a visual analytic system, is a good option for performing bibliometric analysis (Chen, 2004, 2006; Synnestvedt et al., 2005; Chen et al., 2012; Miao et al., 2017). CiteSpace V was used to perform co-citation analysis on authors, and synthesize and visualize the collaborations between countries into a network map which consists of a series of points and lines. In the network map, a point represents a country and a line between two points represents the cooperation relationship (Zheng and Wang, 2019). A wider line indicates a stronger relationship. More importantly, CiteSpace V can help detect the keywords and references with citation bursts. A citation burst has two characteristics, namely, strength and duration (Chen, 2006; Chen et al., 2012). A citation burst indicates increased attention to the underlying work over a certain period of time, which is a key indicator for determining emerging trends (Chen et al., 2014; Liang et al., 2017; Miao et al., 2017). GraphPad Prism 8, which has the basic functions of curve fitting and chart display of biological mathematical statistics, was applied to perform linear regression analysis and analyze the time trend of annual publication outputs.

\section{RESULTS}

\section{Publication Outputs and Time Trend}

A total of 2,986 publications were included in the analysis. The distribution and time trend of annual publication outputs at 


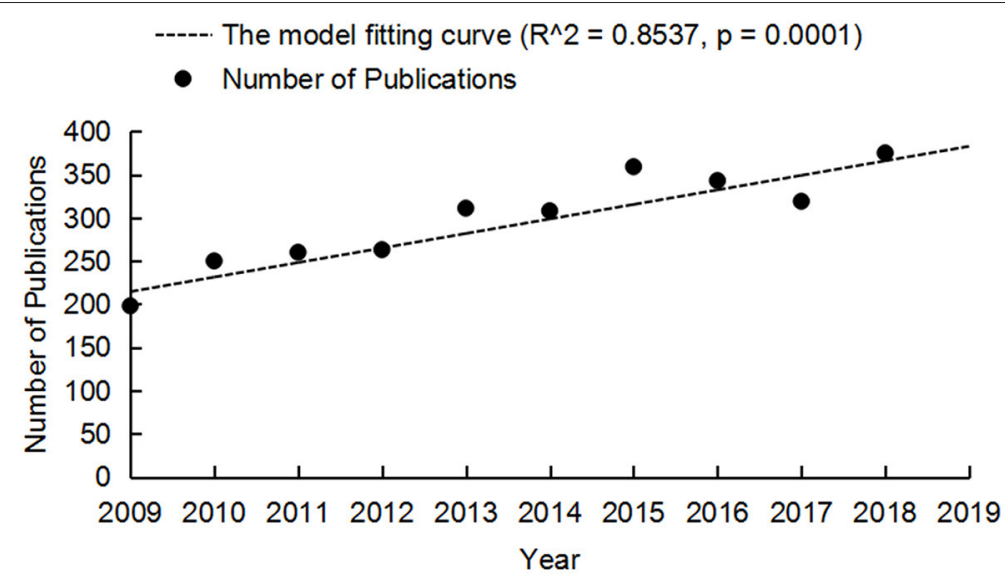

FIGURE 2 | Annual publication outputs and the model fitting curve of time trend of rTMS publications.

TABLE 1 | The top 20 journals that published articles on rTMS research.

\begin{tabular}{|c|c|c|c|c|c|c|}
\hline Rank & Journal title & Count & IF 2018 & Citations WoS & Citations per paper & Country \\
\hline 1 & Brain Stimulation & 199 & 6.919 & 4,566 & 22.94 & USA \\
\hline 2 & PLoS One & 89 & 2.776 & 1,269 & 14.26 & USA \\
\hline 4 & Neuropsychologia & 61 & 2.872 & 1,015 & 16.64 & England \\
\hline 5 & Frontiers in Human Neuroscience & 56 & 2.870 & 986 & 17.61 & Switzerland \\
\hline 8 & Journal of Ect & 49 & 2.280 & 492 & 10.04 & USA \\
\hline 9 & Journal of Affective Disorders & 47 & 4.084 & 799 & 17.00 & Netherlands \\
\hline 10 & Cerebral Cortex & 44 & 5.437 & 1,762 & 40.05 & USA \\
\hline 11 & Neuroimage & 44 & 5.812 & 1,157 & 26.3 & USA \\
\hline 12 & Journal of Neuroscience & 42 & 6.074 & 2,256 & 53.71 & USA \\
\hline 16 & Experimental Brain Research & 30 & 1.878 & 791 & 26.37 & Germany \\
\hline 17 & Frontiers in Neuroscience & 25 & 3.648 & 92 & 3.68 & Switzerland \\
\hline 18 & Scientific Reports & 25 & 4.011 & 82 & 3.28 & England \\
\hline 19 & Human Brain Mapping & 24 & 4.554 & 620 & 25.83 & USA \\
\hline 20 & Journal of Cognitive Neuroscience & 24 & 3.029 & 556 & 23.17 & USA \\
\hline
\end{tabular}

IF, impact factor; WoS, Web of Science.

different time stages are shown in Figure 2. The overall trend is positive, and the publication output was 198 references in 2009 and 375 references in 2018. The time trend of publications indicated a significant correlation $\left(\mathrm{R}^{2}=0.8537, p=0.0001\right)$ between the annual publication outputs and the publication years in the last 10 years.

\section{Distribution by Journal}

The 2,986 publications related to rTMS research were published in 565 scholarly journals. Amongst the top 20 journals shown in Table 1, the average IF was 3.660 (median 3.339, range 1.839-6.919). The journal Brain Stimulation with IF, $2018=$
6.919, published the most number of publications on rTMS research (199) and was cited 4,566 times, followed by PLoS One (publications, 89; IF, $2018=2.776$; citations, 1269), Clinical Neurophysiology (publications, 66; IF, $2018=3.675$; citations, 4,000 ), and Neuropsychologia (publications, 61; IF, $2018=2.872$; citations, 1,015). Clinical Neurophysiology revealed the largest number of citations per paper published (60.61).

\section{Distribution by Country and Institution}

All publications were distributed amongst 43 countries or regions. Amongst the 10 countries shown in Table 2, the USA had the largest number of published papers (764), citations 
TABLE 2 | The top 10 countries of origin of papers in the rTMS research.

\begin{tabular}{llccccc}
\hline Rank & Country & Count & $\begin{array}{c}\text { Citations } \\
\text { WoS }\end{array}$ & $\begin{array}{c}\text { Citations } \\
\text { per paper }\end{array}$ & H-index & $\begin{array}{c}\text { ESI top } \\
\text { paper* }\end{array}$ \\
\hline 1 & USA & 764 & 20,469 & 26.79 & 64 & 17 \\
2 & Germany & 414 & 12,870 & 31.09 & 51 & 11 \\
3 & Italy & 411 & 12,122 & 29.49 & 46 & 5 \\
4 & England & 296 & 10,037 & 33.91 & 46 & 4 \\
5 & Canada & 273 & 8,477 & 31.05 & 41 & 9 \\
6 & China & 266 & 2,712 & 10.20 & 28 & 1 \\
7 & France & 213 & 7,266 & 34.11 & 37 & 6 \\
8 & Australia & 208 & 4,947 & 23.78 & 37 & 3 \\
9 & Japan & 145 & 4,641 & 32.01 & 28 & 3 \\
10 & South Korea & 128 & 1,253 & 9.79 & 19 & 0 \\
\hline
\end{tabular}

ESI, essential science indicators; H-index, Hirsch index; WoS, Web of Science. *There were a total of 33 ESI top papers.

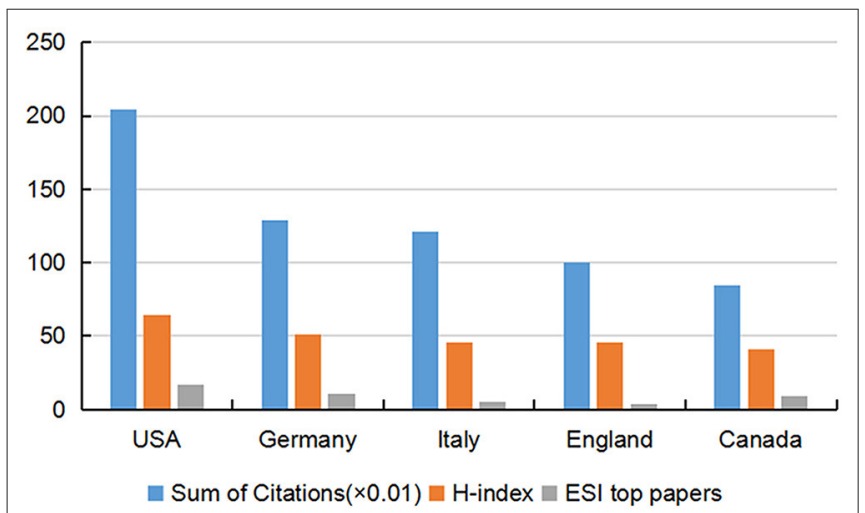

FIGURE 3 | Citation counts $(\times 0.01), \mathrm{H}$-index, and ESI top papers in the top five countries. ESI, essential science indicators; $\mathrm{H}$-index, Hirsch index.

$(20,469)$ and ESI top papers (17) and the highest value of $\mathrm{H}$ index (64). England revealed the largest number of citations per paper (33.91). Figure 3 provides an intuitive comparison of the citations, $\mathrm{H}$-indices and ESI top papers of the top five countries publishing rTMS-related research, and the collaboration network amongst countries/territories is shown in Figure 4. Amongst the 2,986 publications included in this study, $22.67 \%$ were published by the top 10 most prolific institutions. University of Toronto (127) ranked first in the number of publications, followed by Harvard University (112), University College London (83), and Ghent University (58), as presented in Table 3.

\section{Distribution by Author}

Over 9,600 authors contributed to the total output of rTMS research. The publication count in Table 4 reveals that Daskalakis ZJ published 81 papers, ranking first in terms of number of publications, followed by author A (78 publications), author B (65 publications), and Baeken C (51 publications). In terms of cocitation counts, Rossi S (817 citations) ranked first as the most cocited author, followed by author B (594 citations), Wassermann EM (574 citations), and author C (518 citations).

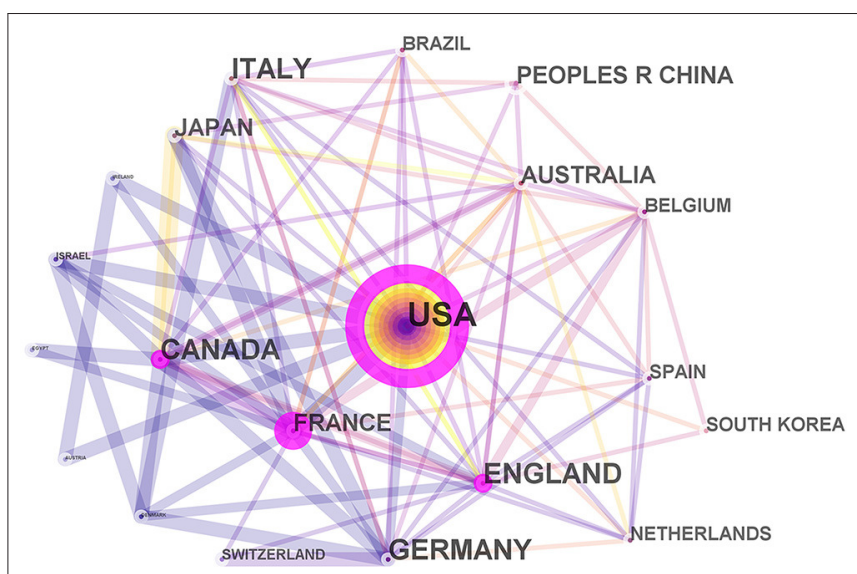

FIGURE 4 | Network map of countries/territories engaged in rTMS research. In the network map, a point represents a country and a line between two points represents the cooperation relationship. A wider line indicates a stronger relationship.

\section{Analysis of References}

The evolution of a knowledge domain can be indicated by references with citation bursts (Synnestvedt et al., 2005; Chen et al., 2014). Table 5 shows the references with the strongest citation bursts during the period 2009-2018. Amongst them, citation bursts by the end of 2018 were led by author D's article published in 2014, which had the strongest burst (71.8868), followed by Rossini et al. (2015) and Berlim et al. (2014).

\section{Analysis of Keywords}

Burst keywords can also be identified as indicators of emerging trends (Chen et al., 2014). Table 6 presents keywords with the strongest citation bursts in this field. The most recent burst keywords were "spinal cord injury," "sham-controlled trial," "recovery," and "functional connectivity."

\section{DISCUSSION}

\section{General Trends of rTMS From 2009 to 2018}

rTMS has received great attention, and research related to the technique has been increasingly performed. It is reasonable to expect a promising future for rTMS based on analyzing the time trend of annual publication outputs.

Amongst the 20 top-performing journals, four journals, namely, Brain Stimulation (IF, $2018=6.919)$, Journal of Neuroscience (IF, $2018=6.074$ ), Neuroimage (IF, $2018=5.812$ ), and Cerebral Cortex (IF, $2018=5.437$ ), had IF scores $>5.000$, and another seven journals had IF scores between 3.000 and 5.000. Approximately 19.09\% (IF, $2018>5.000$, 11.02\%; 5.000 $\geq$ IF, $2018 \geq 3.000,8.07 \%$ ) of the 2,986 publications involved were published in the top 20 journals with high IF (>3.000). In summary, it was challenging to publish papers related to rTMS in high-IF journals.

Amongst the top 10 countries, nine are developed countries and only one (i.e., China) is a developing country. From this point of view, there was still a wide gap between developed and 
TABLE 3 | The top 10 institutions contributed to publications on rTMS research.

\begin{tabular}{|c|c|c|c|c|c|}
\hline Rank & Institution & Count & Rank & Institution & Count \\
\hline 1 & University of Toronto & 127 & 6 & University of São Paulo & 52 \\
\hline 2 & Harvard University & 112 & 7 & McGill University & 50 \\
\hline 3 & University College London & 83 & 8 & Beth Israel Deaconess Medical Center & 49 \\
\hline 4 & Ghent University & 58 & 9 & University of Regensburg & 48 \\
\hline 5 & Monash University & 52 & 10 & Center for Addiction and Mental Health & 46 \\
\hline
\end{tabular}

TABLE 4 | The top 10 authors and co-cited authors in rTMS research.

\begin{tabular}{lllll}
\hline Rank & Author & Count & Co-cited author & Count \\
\hline 1 & Daskalakis ZJ & 81 & Rossi S & 817 \\
2 & Pascual-Leone A & 78 & Fitzgerald PB & 594 \\
3 & Fitzgerald PB & 65 & Wassermann EM & 574 \\
4 & Baeken C & 51 & George MS & 518 \\
5 & George MS & 46 & Fregni F & 477 \\
6 & Langguth B & 46 & Huang YZ & 449 \\
7 & Zangen A & 42 & Lefaucheur JP & 426 \\
8 & Lefaucheur JP & 39 & Chen R & 421 \\
9 & Rothwell JC & 39 & Pascualleone A & 415 \\
10 & Fregni F & 38 & Rossini PM & 327 \\
\hline
\end{tabular}

developing countries in this filed. Although France revealed the largest number of citations per paper (74.6) amongst the top 10 countries publishing rTMS-related research, the USA ranked first in terms of publication count (764), citation count $(20,469)$, and H-index (64). Moreover, the USA had more than half of the ESI top papers $(17,51.52 \%)$, which were hot papers and highly cited papers (Fitzpatrick, 2005; Fu et al., 2011). Therefore, the USA is the leading country in terms of the overall influence in this area.

Information on influential authors can help researchers identify potential collaborators. Authors A, B, C, D, and $\mathrm{E}$ were the most prolific and influential authors in this field, as determined by a comprehensive analysis of numbers of publications and co-citations. Author A suggested that rTMS of the left dorsolateral prefrontal cortex may be a potential treatment for depression (Pascual-Leone et al., 1996). Author B studied the neurobiological mechanisms of the antidepressant effects of rTMS and explored the use of rTMS for depression (Fitzgerald et al., 2006b; Arns et al., 2012; Fitzgerald and Daskalakis, 2012; Noda et al., 2015; Silverstein et al., 2015). Author C confirmed that daily left prefrontal rTMS is safe and effective for treating major depression (MD) (George et al., 2010). Author D showed extensive experience in treating neuropathic pain with rTMS (Lefaucheur, 2006; Lefaucheur et al., 2008b, 2012). Author E studied the effects of non-invasive brain stimulation, including rTMS and transcranial direct current stimulation on psychiatric disorders, pain, and neurological disorders (Lefaucheur et al., 2008a; Miniussi et al., 2008; Zaghi et al., 2009; Brunoni and Fregni, 2011).

\section{Emerging Trends of rTMS}

The evolution of a knowledge domain can be reflected by keywords or references with citation bursts (Fitzpatrick, 2005; Chen, 2006). Therefore, this section analyzes keywords or references showing remarkable bursts by the end of 2018 to reveal emerging trends and provide directions for future research.

\section{Keywords as Indicators of Emerging Trends}

Burst keywords are considered indicators of emerging trends. Four emerging trends in rTMS research were determined according to the most recent keyword bursts; these keywords are listed as follows:

I. Spinal cord injury (SCI): rTMS has emerged as a promising therapeutic technique for SCI patients (de Araújo et al., 2017; Nardone et al., 2017), and the technique has been applied to alleviate some of the main consequences of SCI, including sensory and motor function impairments, spasticity, and neuropathic pain (Tazoe and Perez, 2015; Gunduz et al., 2017). rTMS has also been used in animal experiments on SCI. For example, Krishnan et al. (2019) tested whether rTMS is effective in promoting plasticity and rehabilitation in a rat model of SCI, and their results suggested that rTMS can be used as an early intervention strategy; however, its efficacy and safety in clinical application should be further tested.

II. Sham controlled trial: The type of stimulation is the key point of a sham-controlled trial. Although many studies have included sham-stimulation as control, realistic sham stimulation cannot be guaranteed in studies today (Mennemeier et al., 2009; Lefaucheur et al., 2014). Highquality sham-controlled trials are needed to design a completely blind research. As a preferential option for realistic sham trials, concomitant electrical skin stimulation may be superior to coil angulation and first-generation sham coils (Hosomi et al., 2013; Berlim et al., 2014; Lefaucheur et al., 2014).

III. Recovery: rTMS has a positive impact on functional recovery, such as limb motor recovery in stroke patients; however, optimal rTMS parameters and high-quality evidence require further research (Pollock et al., 2014; Boonzaier et al., 2018; Yang et al., 2018; Xiang et al., 2019).

IV. Functional connectivity: Numerous neuropsychiatric conditions are reportedly coupled with network disturbances (Bassett and Bullmore, 2006, 2017; Grefkes and Fink, 2009, 2011; Frantzidis et al., 2014). As a means of modulating cerebral networks, rTMS not only interferes 
TABLE 5 | References with the strongest citation bursts on rTMS research.

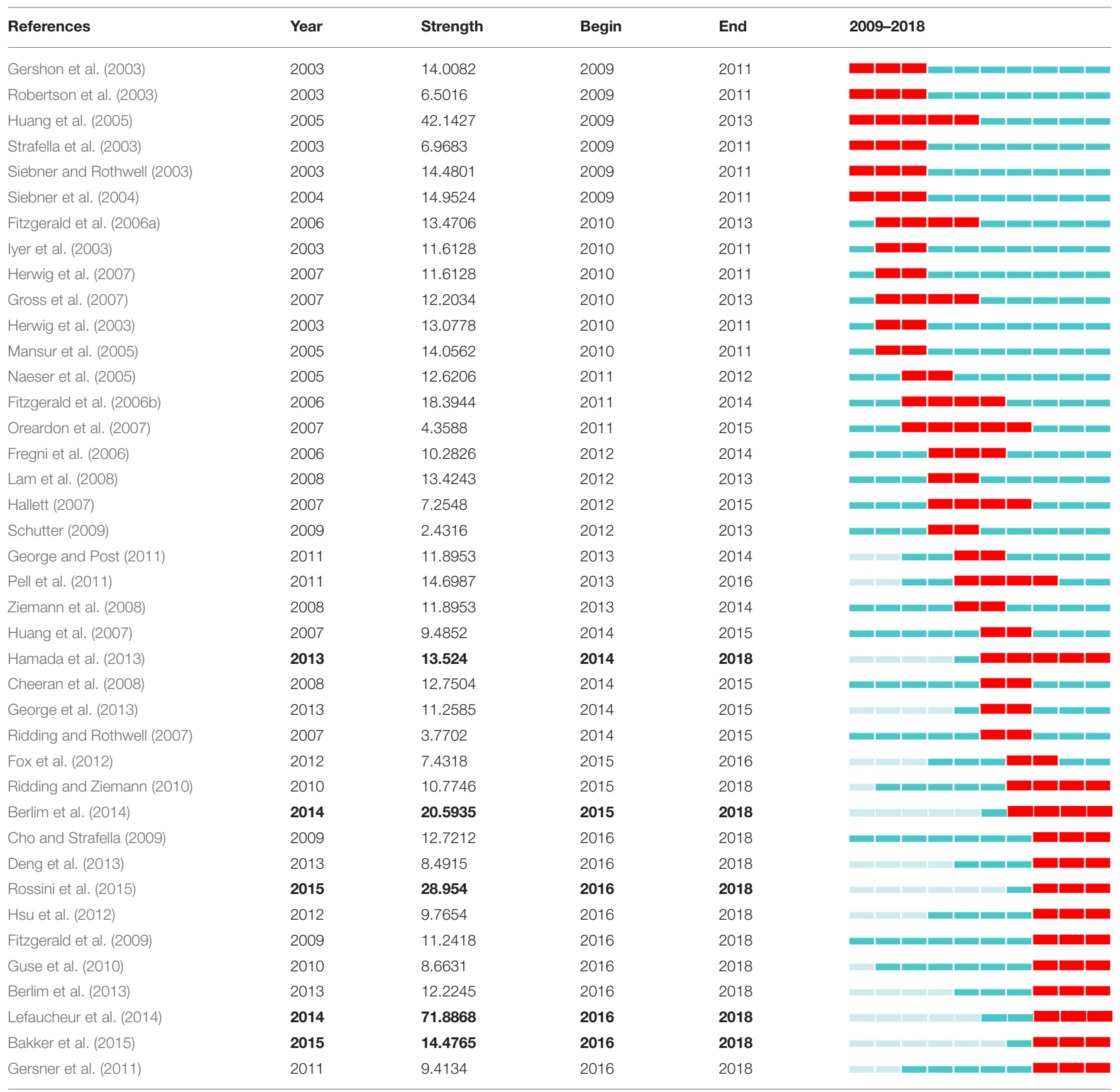

The red bars mean the references cited frequently; the green bars mean the references cited infrequently. A greater strength indicates a higher frequency of citation. The references in bold were reviewed in this study.

with the target cortex but also with those distant and interconnected areas, thereby restoring or increasing functional connectivity (Grefkes and Fink, 2009). Future studies on functional connectivity may facilitate new insights into the pathophysiology of neuropsychiatric conditions and optimize therapeutic strategies of rTMS (Grefkes and Fink, 2011; Krishnan et al., 2019; Kumru et al., 2019; Xiang et al., 2019).

\section{References With Strong Citation Bursts}

References with citation bursts constitute an intellectual base, providing a better understanding of the trends of specific research fields (Fitzpatrick, 2005; Synnestvedt et al., 2005). Instead of discussing all the references with the strongest citation bursts, the following discussions will focus on the top five references by the end of 2018, which are shown in bold in Table 5. 
TABLE 6 | Keywords with the strongest citation bursts of publications on rTMS research.

\begin{tabular}{|c|c|c|c|c|c|}
\hline Keywords & Year & Strength & Begin & End & 2009-2018 \\
\hline Activation & 2009 & 10.9509 & 2009 & 2014 & 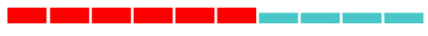 \\
\hline Premotor cortex & 2009 & 8.197 & 2009 & 2010 & שघ \\
\hline Perception & 2009 & 7.4775 & 2009 & 2011 & 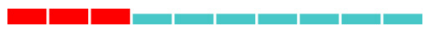 \\
\hline Cortical plasticity & 2009 & 7.346 & 2009 & 2011 & aln \\
\hline Human & 2009 & 6.2637 & 2009 & 2011 & ש \\
\hline Synaptic plasticity & 2009 & 5.9433 & 2009 & 2010 & 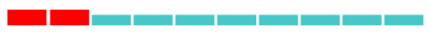 \\
\hline Working memory & 2009 & 3.9615 & 2009 & 2013 & प्रा \\
\hline Paired associative stimulation & 2009 & 3.8581 & 2009 & 2010 & تص \\
\hline Corticospinal excitability & 2009 & 8.7584 & 2010 & 2011 & an \\
\hline Intracortical inhibition & 2009 & 8.5027 & 2010 & 2011 & an \\
\hline Cerebral blood flow & 2009 & 8.0015 & 2010 & 2011 & تصس \\
\hline Treatment & 2009 & 7.5005 & 2010 & 2011 & 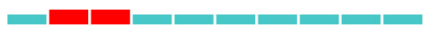 \\
\hline Positron emission tomography & 2009 & 3.6267 & 2010 & 2015 & 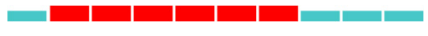 \\
\hline Aphasia & 2009 & 10.4987 & 2011 & 2013 & $\mathrm{Cl}_{\mathrm{b}}$ \\
\hline Tinnitus & 2009 & 8.4556 & 2011 & 2012 & an \\
\hline EEG & 2009 & 5.6254 & 2011 & 2012 & ב \\
\hline Mechanism & 2009 & 3.662 & 2011 & 2012 & 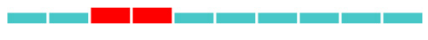 \\
\hline Primary motor cortex & 2009 & 10.6895 & 2012 & 2014 & 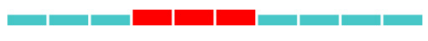 \\
\hline Language & 2009 & 6.7568 & 2012 & 2016 & blv \\
\hline Human brain & 2009 & 9.7966 & 2013 & 2014 & 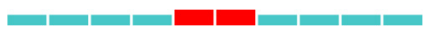 \\
\hline Therapy & 2009 & 6.6686 & 2013 & 2014 & 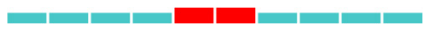 \\
\hline Neuropathic pain & 2009 & 9.4064 & 2014 & 2015 & 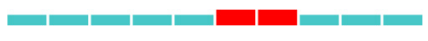 \\
\hline Inhibition & 2009 & 8.5034 & 2014 & 2016 & 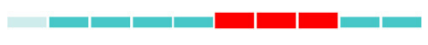 \\
\hline Spinal cord injury & 2009 & 11.0035 & 2015 & 2018 & 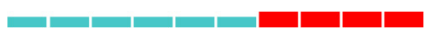 \\
\hline Sham controlled trial & 2009 & 8.5157 & 2015 & 2018 & 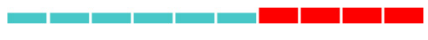 \\
\hline Alzheimers disease & 2009 & 8.105 & 2015 & 2016 & 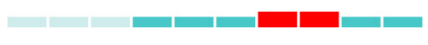 \\
\hline Recovery & 2009 & 5.5658 & 2015 & 2018 & 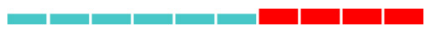 \\
\hline Functional connectivity & 2009 & 4.0777 & 2015 & 2018 & 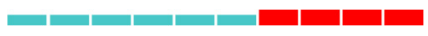 \\
\hline
\end{tabular}

The red bars mean the keywords occurred frequently; the green bars mean the keywords occurred infrequently. A greater strength indicates a higher frequency of occurrence. EEG, electroencephalogram.

As shown in Table 5, a paper by Lefaucheur et al. (2014) revealed the strongest burst by the end of 2018. In this paper, a group of European experts set up evidence-based guidelines on the clinical applications of rTMS in the neurological, ENT (ear, nose, and throat) and psychiatric domains. They also recommended that future technical developments of rTMS concentrate on the creation of new coil shapes and magnetic field geometries and progress of neuronavigation, especially combined with functional imaging and high-resolution EEG, for individualized rTMS treatment.

Rossini et al. (2015) revealed the second strongest citation burst by the end of 2018. The authors updated basic guidelines for clinical application and research on non-invasive stimulation in neuroscience and listed a number of unresolved issues. For example, they described the therapeutic applications of rTMS in depression and neuropathic pain through paradigmatic examples.

The next paper is Berlim et al. (2014), which represented the first meta-analysis to study response, remission, and dropout rates following HF-rTMS for MD. Some practical advice for future studies on rTMS for MD were also proposed. For instance, the authors recommended verification of the clinical utility of the targeted alternative brain region of HF-rTMS for MD.

Bakker et al. (2015) ranked fourth amongst the strongest citation bursts by the end of 2018. In this study, the authors observed 185 depression cases and found that intermittent theta burst stimulation (iTBS) and 5 -fold longer $10 \mathrm{~Hz}$ protocols were comparable in terms of safety, tolerability and efficacy for dorsomedial prefrontal rTMS ( $30 \mathrm{~min}, 10 \mathrm{~Hz}$ vs. 6 min iTBS). Continuation of randomized trials of $10 \mathrm{~Hz}$ and iTBS in future work is recommended.

The burst duration in the study of Hamada et al. (2013) lasted 4 years beginning in 2014. In light of the individual variability existing in the after-effects of rTMS, Hamada et al. examined the effects of rTMS in 56 healthy subjects and provided evidence that individual variations in response to rTMS protocols are due to the neuronal networks activated by each TMS pulse.

To the best our knowledge, this study is the first to assess the trends of rTMS on the basis of literature published from 2009 to 2018 through a bibliometric approach. The study 
provides new insights for clinical and scientific growth and analyzes various aspects of published works. Nevertheless, this work has some limitations. The topic search was only conducted in SCI-EXPANDED of WoS and did not include other databases, such as PubMed and Scopus. Besides, non-English publications, which were few in number and may not change the conclusions, were excluded during retrieval. This study focuses on quantitative analysis but less qualitative analysis.

In conclusion, this study may help investigators discover the publication patterns and emerging trends of rTMS from 2009 to 2018, and presents reference values for the future research and applications of rTMS. The most influential author, institution, journal and country were author A, University of Toronto, Brain Stimulation and the USA, respectively. "Spinal cord injury," "sham-controlled trial," "recovery," and "functional connectivity" may be the latest research frontiers. References with the most recent citation bursts, e.g., Lefaucheur et al. (2014), are worthy of attention and fundamental to emerging trends.

\section{DATA AVAILABILITY STATEMENT}

All datasets generated for this study are included in the article/supplementary material.

\section{REFERENCES}

Alonso, S., Cabrerizo, F. J., and Herrera-Viedma, E. (2009). H-index: a review focused in its variants, computation and standardization for different scientific fields. J. Informetr. 3, 273-289. doi: 10.1016/j.joi.2009.04.001

Arns, M., Drinkenburg, W. H., Fitzgerald, P. B., and Kenemans, J. L. (2012). Neurophysiological predictors of non-response to rTMS in depression. Brain Stimul. 5, 569-576. doi: 10.1016/j.brs.2011.12.003

Bakker, N., Shahab, S., Giacobbe, P., Blumberger, D. M., Daskalakis, Z. J., Kennedy, S. H., et al. (2015). RTMS of the dorsomedial prefrontal cortex for major depression: safety, tolerability, effectiveness, and outcome predictors for $10 \mathrm{~Hz}$ versus intermittent theta-burst stimulation. Brain Stimul. 8, 208-215. doi: $10.1016 /$ j.brs.2014.11.002

Barker, A. T., and Shields, K. (2017). Transcranial magnetic stimulation: basic principles and clinical applications in migraine. Headache 57, 517-524. doi: $10.1111 /$ head.13002

Bassett, D. S., and Bullmore, E. T. (2006). Small-world brain networks. Neuroscientist 12, 512-523. doi: 10.1177/1073858406293182

Bassett, D. S., and Bullmore, E. T. (2017). Small-world brain networks revisited. Neuroscientist 23, 499-516. doi: 10.1177/1073858416667720

Berlim, M. T., van den Eynde, F., and Daskalakis, Z. J. (2013). Clinically meaningful efficacy and acceptability of low-frequency repetitive transcranial magnetic stimulation (rTMS) for treating primary major depression: a meta-analysis of randomized, double-blind and sham-controlled trials. Neuropsychopharmacology. 38, 543-551. doi: 10.1038/npp.2012.237

Berlim, M. T., van den Eynde, F., Tovar-Perdomo, S., and Daskalakis, Z. J. (2014). Response, remission and drop-out rates following high-frequency repetitive transcranial magnetic stimulation (rTMS) for treating major depression: a systematic review and meta-analysis of randomized, double-blind and shamcontrolled trials. Psychol. Med. 44, 225-239. doi: 10.1017/S0033291713000512

Boonzaier, J., van Tilborg, G. A. F., Neggers, S. F. W., and Dijkhuizen, R. M. (2018). Noninvasive brain stimulation to enhance functional recovery after stroke: studies in animal models. Neurorehabil. Neural Repair 32, 927-940. doi: $10.1177 / 1545968318804425$

\section{AUTHOR CONTRIBUTIONS}

This study was conceptualized by K-YZ, G-YD, YL, and X-QW. $\mathrm{K}-\mathrm{YZ}$ contributed to collecting data. Analyzing data and drafting the manuscript were by K-YZ and G-YD. K-YZ, G-YD, YL, and $\mathrm{X}-\mathrm{QW}$ contributed to revising and approving the final version of the manuscript.

\section{FUNDING}

This work was supported by the National Key R\&D Program of China (grant number 2018YFC1314700); Fok Ying-Tong Education Foundation of China (grant number 161092); The scientific and technological research program of the Shanghai Science and Technology Committee (grant number 19080503100); the Shanghai Key Lab of Human Performance (Shanghai University of Sport) (grant number 11DZ2261100); the National Science Foundation of China (grant numbers 81772438, 81572230); Guangzhou Municipal Science and Technology Program (grant number 201803010083).

\section{ACKNOWLEDGMENTS}

We would to thank Linman Weng for assistance with statistical analyses.

Bornmann, L., and Daniel, H. D. (2009). The state of $h$ index research. Is the $h$ index the ideal way to measure research performance? EMBO Rep. 10, 2-6. doi: 10.1038 /embor.2008.233

Brunoni, A. R., and Fregni, F. (2011). Clinical trial design in non-invasive brain stimulation psychiatric research. Int. J. Methods Psychiatr. Res. 20, e19-e30. doi: $10.1002 / \mathrm{mpr} .338$

Cheeran, B., Talelli, P., Mori, F., Koch, G., Suppa, A., Edwards, M., et al. (2008). A common polymorphism in the brain-derived neurotrophic factor gene (BDNF) modulates human cortical plasticity and the response to rTMS. J. Physiol. 586, 5717-5725. doi: 10.1113/jphysiol.2008.159905

Chen, C. M. (2004). Searching for intellectual turning points: progressive knowledge domain visualization. Proc. Natl. Acad. Sci. U.S.A. 101, 5303-5310. doi: 10.1073/pnas.0307513100

Chen, C. M. (2006). CiteSpace II: detecting and visualizing emerging trends and transient patterns in scientific literature. J. Am. Soc. Inf. Sci. Technol. 57, 359-377. doi: 10.1002/asi.20317

Chen, C. M., Dubin, R., and Kim, M. C. (2014). Orphan drugs and rare diseases: a scientometric review (2000-2014). Expert Opin. Orphan Drugs 2, 709-724. doi: $10.1517 / 21678707.2014 .920251$

Chen, C. M., Hu, Z. G., Liu, S. B., and Tseng, H. (2012). Emerging trends in regenerative medicine: a scientometric analysis in CiteSpace. Expert Opin. Biol. Ther. 12, 593-608. doi: 10.1517/14712598.2012.674507

Cho, S. S., and Strafella, A. P. (2009). RTMS of the left dorsolateral prefrontal cortex modulates dopamine release in the ipsilateral anterior cingulate cortex and orbitofrontal cortex. PLoS ONE 4:e6725. doi: 10.1371/journal.pone.0006725

Cirillo, G., Di Pino, G., Capone, F., Ranieri, F., Florio, L., Todisco, V., et al. (2017). Neurobiological after-effects of non-invasive brain stimulation. Brain Stimul. 10, 1-18. doi: 10.1016/j.brs.2016.11.009

da Silva, J. A. T., and Dobranszki, J. (2018). Multiple versions of the h-index: cautionary use for formal academic purposes. Scientometrics 115, 1107-1113. doi: 10.1007/s11192-018-2680-3

Dayan, E., Censor, N., Buch, E. R., Sandrini, M., and Cohen, L. G. (2013). Noninvasive brain stimulation: from physiology to network dynamics and back. Nat. Neurosci. 16, 838-844. doi: 10.1038/nn.3422 
de Araújo, A. V. L., Barbosa, V. R. N., Galdino, G. S., Fregni, F., Massetti, T., Fontes, S. L., et al. (2017). Effects of high-frequency transcranial magnetic stimulation on functional performance in individuals with incomplete spinal cord injury: study protocol for a randomized controlled trial. Trials 18:522. doi: 10.1186/s13063-017-2280-1

Deng, Z. D., Lisanby, S. H., and Peterchev, A. V. (2013). Electric field depth-focality tradeoff in transcranial magnetic stimulation: simulation comparison of 50 coil designs. Brain Stimul. 6, 1-13. doi: 10.1016/j.brs.2012.02.005

Ellegaard, O., and Wallin, J. A. (2015). The bibliometric analysis of scholarly production: how great is the impact? Scientometrics 105, 1809-1831. doi: 10.1007/s11192-015-1645-Z

Fitzgerald, P. B., Benitez, J., de Castella, A., Daskalakis, Z. J., Brown, T. L., and Kulkarni, J. (2006a). A randomized, controlled trial of sequential bilateral repetitive transcranial magnetic stimulation for treatment-resistant depression. Am. J. Psychiatry 163, 88-94. doi: 10.1176/appi.ajp.163.1.88

Fitzgerald, P. B., and Daskalakis, Z. J. (2012). A practical guide to the use of repetitive transcranial magnetic stimulation in the treatment of depression. Brain Stimul. 5, 287-296. doi: 10.1016/j.brs.2011.03.006

Fitzgerald, P. B., Fountain, S., and Daskalakis, Z. J. (2006b). A comprehensive review of the effects of rTMS on motor cortical excitability and inhibition. Clin. Neurophysiol. 117, 2584-2596. doi: 10.1016/j.clinph.2006.06.712

Fitzgerald, P. B., Hoy, K., McQueen, S., Maller, J. J., Herring, S., Segrave, R., et al. (2009). A randomized trial of rTMS targeted with MRI based neuro-navigation in treatment-resistant depression. Neuropsychopharmacology 34, 1255-1262. doi: $10.1038 / \mathrm{npp} .2008 .233$

Fitzpatrick, R. B. (2005). Essential science indicators. Med. Ref. Serv. Q. 24, 67-78. doi: 10.1300/J115v24n04_05

Fox, M. D., Buckner, R. L., White, M. P., Greicius, M. D., and Pascual-Leone, A. (2012). Efficacy of transcranial magnetic stimulation targets for depression is related to intrinsic functional connectivity with the subgenual cingulate. Biol. Psychiatry 72, 595-603. doi: 10.1016/j.biopsych.2012.04.028

Frantzidis, C. A., Vivas, A. B., Tsolaki, A., Klados, M. A., Tsolaki, M., and Bamidis, P. D. (2014). Functional disorganization of small-world brain networks in mild alzheimer's disease and amnestic mild cognitive impairment: an EEG study using relative wavelet entropy (RWE). Front. Aging Neurosci. 6:224. doi: 10.3389/fnagi.2014.00224

Fregni, F., Boggio, P. S., Valle, A. C., Rocha, R. R., Duarte, J., Ferreira, M. J., et al. (2006). A sham-controlled trial of a 5-day course of repetitive transcranial magnetic stimulation of the unaffected hemisphere in stroke patients. Stroke 37, 2115-2122. doi: 10.1161/01.STR.0000231390.58967.6b

Fu, H. Z., Chuang, K. Y., Wang, M. H., and Ho, Y. S. (2011). Characteristics of research in China assessed with essential science indicators. Scientometrics 88 , 841-862. doi: 10.1007/s11192-011-0416-8

George, M. S., and Aston-Jones, G. (2010). Noninvasive techniques for probing neurocircuitry and treating illness: vagus nerve stimulation (VNS), transcranial magnetic stimulation (TMS) and transcranial direct current stimulation (tDCS). Neuropsychopharmacology 35, 301-316. doi: 10.1038/npp.2009.87

George, M. S., Lisanby, S. H., Avery, D., McDonald, W. M., Durkalski, V., Pavlicova, M., et al. (2010). Daily left prefrontal transcranial magnetic stimulation therapy for major depressive disorder: a shamcontrolled randomized trial. Arch. Gen. Psychiatry 67, 507-516. doi: 10.1001/archgenpsychiatry.2010.46

George, M. S., and Post, R. M. (2011). Daily left prefrontal repetitive transcranial magnetic stimulation for acute treatment of medication-resistant depression. Am. J. Psychiatry 168, 356-364. doi: 10.1176/appi.ajp.2010.10060864

George, M. S., Taylor, J. J., and Short, E. B. (2013). The expanding evidence base for rTMS treatment of depression. Curr. Opin. Psychiatry 26, 13-18. doi: 10.1097/YCO.0b013e32835ab46d

Gershon, A. A., Dannon, P. N., and Grunhaus, L. (2003). Transcranial magnetic stimulation in the treatment of depression. Am. J. Psychiatry 160, 835-845. doi: 10.1176/appi.ajp.160.5.835

Gersner, R., Kravetz, E., Feil, J., Pell, G., and Zangen, A. (2011). Long-term effects of repetitive transcranial magnetic stimulation on markers for neuroplasticity: differential outcomes in anesthetized and awake animals. J. Neurosci. 31, 7521-7526. doi: 10.1523/JNEUROSCI.6751-10.2011

Grefkes, C., and Fink, G. R. (2009). Functional neuroimaging and neuromodulation: effects of transcranial magnetic stimulation on cortical networks in healthy subjects and patients. Klin. Neurophysiol. 40, 239-247. doi: 10.1055/s-0029-1242733

Grefkes, C., and Fink, G. R. (2011). Reorganization of cerebral networks after stroke: new insights from neuroimaging with connectivity approaches. Brain 134, 1264-1276. doi: 10.1093/brain/awr033

Gross, M., Nakamura, L., Pascual-Leone, A., and Fregni, F. (2007). Has repetitive transcranial magnetic stimulation (rTMS) treatment for depression improved? a systematic review and meta-analysis comparing the recent vs. the earlier rTMS studies. Acta Psychiatr. Scand. 116, 165-173. doi: 10.1111/j.1600-0447.2007.01049.x

Gunduz, A., Rothwell, J., Vidal, J., and Kumru, H. (2017). Non-invasive brain stimulation to promote motor and functional recovery following spinal cord injury. Neural Regen. Res. 12, 1933-1938. doi: 10.4103/1673-5374.221143

Guse, B., Falkai, P., and Wobrock, T. (2010). Cognitive effects of high-frequency repetitive transcranial magnetic stimulation: a systematic review. J. Neural Transm. 117, 105-122. doi: 10.1007/s00702-009-0333-7

Hallett, M. (2007). Transcranial magnetic stimulation: a primer. Neuron 55, 187-199. doi: 10.1016/j.neuron.2007.06.026

Hamada, M., Murase, N., Hasan, A., Balaratnam, M., and Rothwell, J. C. (2013). The role of interneuron networks in driving human motor cortical plasticity. Cereb. Cortex 23, 1593-1605. doi: 10.1093/cercor/bhs147

Hao, Z. L., Wang, D. R., Zeng, Y., and Liu, M. (2013). Repetitive transcranial magnetic stimulation for improving function after stroke. Cochrane Database Syst. Rev. CD008862. doi: 10.1002/14651858.CD010820.pub2008862

Herwig, U., Fallgatter, A. J., Höppner, J., Eschweiler, G. W., Kron, M., Hajak, G., et al. (2007). Antidepressant effects of augmentative transcranial magnetic stimulation: randomised multicentre trial. Br. J. Psychiatry 191, 441-448. doi: 10.1192/bjp.bp.106.034371

Herwig, U., Satrapi, P., and Schönfeldt-Lecuona, C. (2003). Using the international 10-20 EEG system for positioning of transcranial magnetic stimulation. Brain Topogr. 16, 95-99. doi: 10.1023/B:BRAT.0000006333.93597.9d

Hirsch, J. E. (2005). An index to quantify an individual's scientific research output. Proc. Natl. Acad. Sci. U.S.A. 102, 16569-16572. doi: 10.1073/pnas.0507655102

Hosomi, K., Shimokawa, T., Ikoma, K., Nakamura, Y., Sugiyama, K., Ugawa, Y., et al. (2013). Daily repetitive transcranial magnetic stimulation of primary motor cortex for neuropathic pain: a randomized, multicenter, double-blind, crossover, sham-controlled trial. Pain 154, 1065-1072. doi: 10.1016/j.pain.2013.03.016

Hsu, W. Y., Cheng, C. H., Liao, K. K., Lee, I. H., and Lin, Y. Y. (2012). Effects of repetitive transcranial magnetic stimulation on motor functions in patients with stroke: a meta-analysis. Stroke 43, 1849-1857. doi: 10.1161/STROKEAHA.111.649756

Huang, Y. Z., Chen, R. S., Rothwell, J. C., and Wen, H. Y. (2007). The aftereffect of human theta burst stimulation is NMDA receptor dependent. Clin. Neurophysiol. 118, 1028-1032. doi: 10.1016/j.clinph.2007.01.021

Huang, Y. Z., Edwards, M. J., Rounis, E., Bhatia, K. P., and Rothwell, J. C. (2005). Theta burst stimulation of the human motor cortex. Neuron 45, 201-206. doi: 10.1016/j.neuron.2004.12.033

Hummel, F. C., and Cohen, L. G. (2006). Non-invasive brain stimulation: a new strategy to improve neurorehabilitation after stroke? Lancet Neurol. 5, 708-712. doi: 10.1016/S1474-4422(06)70525-7

Iyer, M. B., Schleper, N., and Wassermann, E. M. (2003). Priming stimulation enhances the depressant effect of low-frequency repetitive transcranial magnetic stimulation. J. Neurosci. 23, 10867-10872. doi: 10.1523/JNEUROSCI.23-34-10867.2003

Jansen, J. M., van den Heuvel, O. A., van der Werf, Y. D., de Wit, S. J., Veltman, D. J., van den Brink, W., et al. (2019). The effect of highfrequency repetitive transcranial magnetic stimulation on emotion processing, reappraisal, and craving in alcohol use disorder patients and healthy controls: a functional magnetic resonance imaging study. Front. Psychiatry 10:272. doi: 10.3389/fpsyt.2019.00272

Klooster, D. C., de Louw, A. J., Aldenkamp, A. P., Besseling, R. M., Mestrom, R. M., Carrette, S., et al. (2016). Technical aspects of neurostimulation: focus on equipment, electric field modeling, and stimulation protocols. Neurosci. Biobehav. Rev. 65, 113-141. doi: 10.1016/j.neubiorev.2016.02.016

Krishnan, V. S., Shin, S. S., Belegu, V., Celnik, P., Reimers, M., Smith, K. R., and Pelled, G. (2019). Multimodal evaluation of TMS - induced somatosensory 
plasticity and behavioral recovery in rats with contusion spinal cord injury. Front. Neurosci. 13:387. doi: 10.3389/fnins.2019.00387

Kumru, H., Kofler, M., Valls-Sole, J., and Vidal, J. (2019). Brainstem reflex excitability after high-frequency repetitive transcranial magnetic stimulation in healthy and spinal cord injury subjects. Brain Res. Bull. 147, 86-91. doi: 10.1016/j.brainresbull.2019.02.002

Kwon, T. G., Park, E., Kang, C., Chang, W. H., and Kim, Y. H. (2016). The effects of combined repetitive transcranial magnetic stimulation and transcranial direct current stimulation on motor function in patients with stroke. Restor. Neurol. Neurosci. 34, 915-923. doi: 10.3233/RNN-160654

Lam, R. W., Chan, P., Wilkins-Ho, M., and Yatham, L. N. (2008). Repetitive transcranial magnetic stimulation for treatment-resistant depression: a systematic review and metaanalysis. Can. J. Psychiatry 53, 621-631. doi: $10.1177 / 070674370805300909$

Lefaucheur, J. P. (2006). The use of repetitive transcranial magnetic stimulation (rTMS) in chronic neuropathic pain. Neurophysiol. Clin. 36, 117-124. doi: 10.1016/j.neucli.2006.08.002

Lefaucheur, J. P., André-Obadia, N., Antal, A., Ayache, S. S., Baeken, C., Benninger, D. H., et al. (2014). Evidence-based guidelines on the therapeutic use of repetitive transcranial magnetic stimulation (rTMS). Clin. Neurophysiol. 125, 2150-2206. doi: 10.1016/j.clinph.2014.05.021

Lefaucheur, J. P., Antal, A., Ahdab, R., Ciampi de Andrade, D., Fregni, F., Khedr, E. M., et al. (2008a). The use of repetitive transcranial magnetic stimulation (rTMS) and transcranial direct current stimulation (tDCS) to relieve pain. Brain Stimul. 1, 337-344. doi: 10.1016/j.brs.2008.07.003

Lefaucheur, J. P., Ayache, S. S., Sorel, M., Farhat, W. H., Zouari, H. G., Ciampi de Andrade, D., et al. (2012). Analgesic effects of repetitive transcranial magnetic stimulation of the motor cortex in neuropathic pain: influence of theta burst stimulation priming. Eur. J. Pain 16, 1403-1413. doi: 10.1002/j.1532-2149.2012.00150.x

Lefaucheur, J. P., Drouot, X., Ménard-Lefaucheur, I., Keravel, Y., and Nguyen, J. P. (2008b). Motor cortex rTMS in chronic neuropathic pain: pain relief is associated with thermal sensory perception improvement. J. Neurol. Neurosurg. Psychiatr. 79, 1044-1049. doi: 10.1136/jnnp.2007.135327

Liang, Y. D., Li, Y., Zhao, J., Wang, X. Y., Zhu, H. Z., and Chen, X. H. (2017). Study of acupuncture for low back pain in recent 20 years: a bibliometric analysis via CiteSpace. J. Pain Res. 10, 951-964. doi: 10.2147/JPR.S132808

Liao, H. C., Tang, M., Luo, L., Li, C. Y., Chiclana, F., and Zeng, X. J. (2018). A bibliometric analysis and visualization of medical big data research. Sustainability 10:166. doi: 10.3390/su10010166

Lowe, C. J., Vincent, C., and Hall, P. A. (2017). Effects of noninvasive brain stimulation on food cravings and consumption: a meta-analytic review. Psychosom. Med. 79, 2-13. doi: 10.1097/PSY.0000000000000368

Lucena, M. F. G., Teixeira, P. E. P., Bonin Pinto, C., and Fregni, F. (2019). Top 100 cited noninvasive neuromodulation clinical trials. Expert Rev. Med. Dev. 16, 451-466. doi: 10.1080/17434440.2019.1615440

Maeda, F., Keenan, J. P., Tormos, J. M., Topka, H., and Pascual-Leone, A. (2000). Modulation of corticospinal excitability by repetitive transcranial magnetic stimulation. Clin. Neurophysiol. 111, 800-805. doi: 10.1016/S1388-2457(99)00323-5

Mansur, C. G., Fregni, F., Boggio, P. S., Riberto, M., Gallucci-Neto, J., Santos, C. M., et al. (2005). A sham stimulation-controlled trial of rTMS of the unaffected hemisphere in stroke patients. Neurology 64, 1802-1804. doi: 10.1212/01.WNL.0000161839.38079.92

McLean, A. L. (2019). Publication trends in transcranial magnetic stimulation: a 30-year panorama. Brain Stimul. 12, 619-627. doi: 10.1016/j.brs.2019.01.002

Mennemeier, M., Triggs, W., Chelette, K., Woods, A., Kimbrell, T., and Dornhoffer, J. (2009). Sham transcranial magnetic stimulation using electrical stimulation of the scalp. Brain Stimul. 2, 168-173. doi: 10.1016/j.brs.2009.02.002

Miao, Y., Xu, S. Y., Chen, L. S., Liang, G. Y., Pu, Y. P., and Yin, L. H. (2017). Trends of long noncoding RNA research from 2007 to 2016: a bibliometric analysis. Oncotarget 8, 83114-83127. doi: 10.18632/oncotarget.20851

Miniussi, C., Cappa, S. F., Cohen, L. G., Floel, A., Fregni, F., Nitsche, M. A., et al. (2008). Efficacy of repetitive transcranial magnetic stimulation/transcranial direct current stimulation in cognitive neurorehabilitation. Brain Stimul. 1, 326-336. doi: 10.1016/j.brs.2008.07.002
Miniussi, C., Harris, J. A., and Ruzzoli, M. (2013). Modelling non-invasive brain stimulation in cognitive neuroscience. Neurosci. Biobehav. Rev. 37, 1702-1712. doi: 10.1016/j.neubiorev.2013.06.014

Naeser, M. A., Martin, P. I., Nicholas, M., Baker, E. H., Seekins, H., Kobayashi, M., et al. (2005). Improved picture naming in chronic aphasia after TMS to part of right broca's area: an open-protocol study. Brain Lang. 93, 95-105. doi: 10.1016/j.bandl.2004.08.004

Nardone, R., Langthaler, P. B., Orioli, A., Höller, P., Höller, Y., Frey, V. N., et al. (2017). Effects of intermittent theta burst stimulation on spasticity after spinal cord injury. Restor. Neurol. Neurosci. 35, 287-294. doi: 10.3233/RNN-160701

Noda, Y., Silverstein, W. K., Barr, M. S., Vila-Rodriguez, F., Downar, J., Rajji, T. K., et al. (2015). Neurobiological mechanisms of repetitive transcranial magnetic stimulation of the dorsolateral prefrontal cortex in depression: a systematic review. Psychol. Med. 45, 3411-3432. doi: 10.1017/S0033291715001609

Oelrich, B., Peters, R., and Jung, K. (2007). A bibliometric evaluation of publications in urological journals among European Union countries between 2000-2005. Eur. Urol. 52, 1238-1248. doi: 10.1016/j.eururo.2007.06.050

Oreardon, J. P., Solvason, H. B., Janicak, P. G., Sampson, S., Isenberg, K. E., Nahas, Z., et al. (2007). Efficacy and safety of transcranial magnetic stimulation in the acute treatment of major depression: a multisite randomized controlled trial. Biol. Psychiatry 62, 1208-1216. doi: 10.1016/j.biopsych.2007.01.018

Pascual-Leone, A., Rubio, B., Pallardó, F., and Catalá, M. D. (1996). Rapid-rate transcranial magnetic stimulation of left dorsolateral prefrontal cortex in drugresistant depression. Lancet 348, 233-237. doi: 10.1016/S0140-6736(96)01219-6

Pell, G. S., Roth, Y., and Zangen, A. (2011). Modulation of cortical excitability induced by repetitive transcranial magnetic stimulation: influence of timing and geometrical parameters and underlying mechanisms. Prog. Neurobiol. 93, 59-98. doi: 10.1016/j.pneurobio.2010.10.003

Pollock, A., Farmer, S. E., Brady, M. C., Langhorne, P., Mead, G. E., Mehrholz, J., et al. (2014). Interventions for improving upper limb function after stroke. Cochrane Database Syst. Rev. CD010820. doi: 10.1002/14651858.CD010820.pub2

Reithler, J., Peters, J. C., and Sack, A. T. (2011). Multimodal transcranial magnetic stimulation: using concurrent neuroimaging to reveal the neural network dynamics of noninvasive brain stimulation. Prog. Neurobiol. 94, 149-165. doi: 10.1016/j.pneurobio.2011.04.004

Ridding, M. C., and Rothwell, J. C. (2007). Is there a future for therapeutic use of transcranial magnetic stimulation? Nat. Rev. Neurosci. 8, 559-567. doi: $10.1038 / \mathrm{nrn} 2169$

Ridding, M. C., and Ziemann, U. (2010). Determinants of the induction of cortical plasticity by non-invasive brain stimulation in healthy subjects. J. Physiol. 588, 2291-2304. doi: 10.1113/jphysiol.2010.190314

Robertson, E. M., Théoret, H., and Pascual-Leone, A. (2003). Studies in cognition: the problems solved and created by transcranial magnetic stimulation. J. Cogn. Neurosci. 15, 948-960. doi: 10.1162/089892903770007344

Rossini, P. M., Burke, D., Chen, R., Cohen, L. G., Daskalakis, Z., Di Iorio, R., et al. (2015). Non-invasive electrical and magnetic stimulation of the brain, spinal cord, roots and peripheral nerves: basic principles and procedures for routine clinical and research application. An updated report from an IFCN committee. Clin. Neurophysiol. 126, 1071-1107. doi: 10.1016/j.clinph.2015.02.001

Schutter, D. J. (2009). Antidepressant efficacy of high-frequency transcranial magnetic stimulation over the left dorsolateral prefrontal cortex in doubleblind sham-controlled designs: a meta-analysis. Psychol. Med. 39, 65-75. doi: $10.1017 /$ S0033291708003462

Siebner, H. R., Lang, N., Rizzo, V., Nitsche, M. A., Paulus, W., Lemon, R. N., et al. (2004). Preconditioning of low-frequency repetitive transcranial magnetic stimulation with transcranial direct current stimulation: evidence for homeostatic plasticity in the human motor cortex. J. Neurosci. 24, 3379-3385. doi: 10.1523/JNEUROSCI.5316-03.2004

Siebner, H. R., and Rothwell, J. (2003). Transcranial magnetic stimulation: new insights into representational cortical plasticity. Exp. Brain Res. 148, 1-16. doi: 10.1007/s00221-002-1234-2

Silverstein, W. K., Noda, Y., Barr, M. S., Vila-Rodriguez, F., Rajji, T. K., Fitzgerald, P. B., et al. (2015). Neurobiological predictors of response to dorsolateral prefrontal cortex repetitive transcranial magnetic stimulation in depression: a systematic review. Depress. Anxiety 32, 871-891. doi: 10.1002/da. 22424 
Strafella, A. P., Paus, T., Fraraccio, M., and Dagher, A. (2003). Striatal swap release induced by repetitive transcranial magnetic stimulation of the human motor cortex. Brain 126, 2609-2615. doi: 10.1093/brain/awg268

Synnestvedt, M. B., Chen, C., and Holmes, J. H. (2005). CiteSpace II: visualization and knowledge discovery in bibliographic databases. AMIA Annu. Symp. Proc. $2005,724-728$

Tazoe, T., and Perez, M. A. (2015). Effects of repetitive transcranial magnetic stimulation on recovery of function after spinal cord injury. Arch. Phys. Med. Rehabil. 96, S145-S155. doi: 10.1016/j.apmr.2014.07.418

Terranova, C., Rizzo, V., Cacciola, A., Chillemi, G., Calamuneri, A., Milardi, D., et al. (2019). Is there a future for non-invasive brain stimulation as a therapeutic tool? Front. Neurol. 9:1146. doi: 10.3389/fneur.2018. 01146

Thompson, D. F., and Walker, C. K. (2015). A descriptive and historical review of bibliometrics with applications to medical sciences. Pharmacotherapy 35, 551-559. doi: 10.1002/phar.1586

Wang, X. Q., Peng, M. S., Weng, L. M., Zheng, Y. L., Zhang, Z. J., and Chen, P. J. (2019). Bibliometric study of the comorbidity of pain and depression research. Neural Plast. 2019:1657498. doi: 10.1155/2019/1657498

Xiang, H. F., Sun, J., Tang, X., Zeng, K. B., and Wu, X. S. (2019). The effect and optimal parameters of repetitive transcranial magnetic stimulation on motor recovery in stroke patients: a systematic review and meta-analysis of randomized controlled trials. Clin. Rehabil. 33, 847-864. doi: 10.1177/0269215519829897

Yang, C. X., Guo, Z. W., Peng, H. T., Xing, G. Q., Chen, H. P., McClure, M. A., et al. (2018). Repetitive transcranial magnetic stimulation therapy for motor recovery in parkinson's disease: a Meta-analysis. Brain Behav. 8:e01132. doi: $10.1002 /$ brb3.1132
Yeung, A. W. K., Goto, T. K., and Leung, W. K. (2017). A bibliometric review of research trends in neuroimaging. Curr. Sci. 112, 725-734. doi: 10.18520/cs/v112/i04/725-734

Zaghi, S., Heine, N., and Fregni, F. (2009). Brain stimulation for the treatment of pain: a review of costs, clinical effects, and mechanisms of treatment for three different central neuromodulatory approaches. J. Pain Manag. 2, 339-352.

Zheng, K. Y., and Wang, X. Q. (2019). Publications on the association between cognitive function and pain from 2000 to 2018: a bibliometric analysis using CiteSpace. Med. Sci. Monit. 25, 8940-8951. doi: 10.12659/MSM.917742

Ziemann, U., Paulus, W., Nitsche, M. A., Pascual-Leone, A., Byblow, W. D., Berardelli, A., et al. (2008). Consensus: motor cortex plasticity protocols. Brain Stimul. 1, 164-182. doi: 10.1016/j.brs.2008.06.006

Zorzo, C., Higarza, S. G., Méndez, M., Martínez, J. A., Pernía, A. M., and Arias, J. L. (2019). High frequency repetitive transcranial magnetic stimulation improves neuronal activity without affecting astrocytes and microglia density. Brain Res. Bull. 150, 13-20. doi: 10.1016/j.brainresbull.2019.05.004

Conflict of Interest: The authors declare that the research was conducted in the absence of any commercial or financial relationships that could be construed as a potential conflict of interest.

Copyright (c) 2020 Zheng, Dai, Lan and Wang. This is an open-access article distributed under the terms of the Creative Commons Attribution License (CC BY). The use, distribution or reproduction in other forums is permitted, provided the original author(s) and the copyright owner(s) are credited and that the original publication in this journal is cited, in accordance with accepted academic practice. No use, distribution or reproduction is permitted which does not comply with these terms. 